\title{
THE SPITZER c2d SURVEY OF NEARBY DENSE CORES: JET AND MOLECULAR OUTFLOW ASSOCIATED WITH A YOUNG STELLAR OBJECT IN CORE A OF L1251
}

\author{
Jeong-Eun Lee ${ }^{1}$, Ho-Gyu Lee ${ }^{2}$, Jong-Ho Shinn ${ }^{3}$, Michael M. Dunham ${ }^{4}$, Il-Suk Kim ${ }^{1}$, Chang Hee Kim ${ }^{5}$, \\ Tyler L. Bourke ${ }^{6}$, Neal J. Evans ${ }^{4}$, And YunheE Choi ${ }^{1}$ \\ ${ }^{1}$ Department of Astronomy and Space Science, Astrophysical Research Center for the Structure and Evolution of the Cosmos, Sejong University, Seoul 143-747, \\ Republic of Korea; jelee@sejong.ac.kr \\ 2 Department of Astronomy and Astrophysics, University of Toronto, Toronto, ON, M5S 3H4, Canada; tohogyu @ gmail.com \\ ${ }^{3}$ Korea Astronomy and Space Science Institute, Daejeon 305-348, Republic of Korea \\ ${ }^{4}$ Astronomy Department, The University of Texas at Austin, 1 University Station C1400, Austin, TX 78712-0259, USA; mdunham@astro.as.utexas.edu, \\ nje@astro.as.utexas.edu \\ ${ }^{5}$ Department of Physics and Astronomy, Seoul National University, 599 Gwanangno, Gwanak-gu, Seoul 151-747, Republic of Korea \\ ${ }^{6}$ Smithsonian Astrophysical Observatory, 60 Garden Street, Cambridge, MA 02138, USA; tbourke@ cfa.harvard.edu \\ Received 2009 September 27; accepted 2009 December 15; published 2010 January 5
}

\begin{abstract}
A long infrared jet has been discovered by the Spitzer c2d Legacy Program in core A of L1251. It is associated with a very embedded Class 0 object with an accretion luminosity of about $0.9 L_{\odot}$ derived by radiative transfer model fitting to the observed spectral energy distribution. Comparing the observed Infrared Array Camera colors along the infrared jet with those calculated from a model of an admixture of gas with a power-law temperature distribution indicates that the jet is possibly created by a paraboloidal bow shock propagating into the ambient medium of $n\left(\mathrm{H}_{2}\right)=10^{5} \mathrm{~cm}^{-3}$. In addition, the variation of the power-law index along the jet suggests that the portion of hot gas decreases with distance from the jet engine. The molecular outflow in this region has been mapped for the first time using $\mathrm{CO}$ data. From the calculated outflow momentum flux, a very strong lower limit to the average accretion luminosity is $3.6 \frac{\sin i}{\cos ^{3} i} L_{\odot}$, indicative of a decrease in the accretion rate with time. Key words: ISM: individual objects (L1251) - stars: formation
\end{abstract}

\section{INTRODUCTION}

Stars form out of the collapse of dense molecular gas cores. However, this process is complex, and the details are far from understood. Young stellar objects (YSOs) and their immediate surroundings are expected to evolve significantly throughout star formation. For example, YSOs will evolve in luminosity and temperature, as their masses grow and they evolve toward the main sequence (e.g., Myers et al. 1998). In addition, the associated gas will evolve dynamically. As collapse proceeds, gas flows from cores through disks and onto protostars, driving jets and outflows in the process. These outflows are a crucial mechanism by which angular momentum is removed from a star-forming system where accretion occurs through a rotating disk. Therefore, an outflow is a good tracer of star formation, especially when the central protostar is deeply embedded.

Large molecular outflows have been mapped in (sub)millimeter lines of CO (Fukui 1989; Morgan et al. 1991), which trace the matter cooled down after being shocked. However, more recently, shocked material such as warm molecular hydrogen produces rovibrational or pure rotational transitions at infrared wavelengths. The Infrared Spectrograph (IRS) aboard the Spitzer Space Telescope has been used to map the shocked $\mathrm{H}_{2}$ line emission (Neufeld et al. 2006; Maret et al. 2009). Recently Neufeld \& Yuan (2008) showed that the Spitzer Infrared Array Camera (IRAC) intensities were dominated by warm molecular hydrogen emission in the supernova remnant IC 443. In addition, Ybarra \& Lada (2009) also showed that IRAC colors could be used to study the properties of the hot, shocked gas components in HH54.

The Spitzer Legacy Program "From Molecular Cores to Planet Forming Disks" (c2d; Evans et al. 2003) discovered a new jet feature on the western edge of L1251 (core A in Sato et al. 1994), whose distance is 300( \pm 50$)$ pc (Kun \& Prusti 1993).
No outflows were previously detected in this region although there are three IRAS sources (Kun \& Prusti 1993; Sato et al. 1994). No optical jets or Herbig-Haro (HH) objects have been reported in this region, probably due to the high optical depth. The discovered infrared jet is the longest $(\sim 1 \mathrm{pc})$ except $\mathrm{HH}$ 111 , which is onesided and $\sim 8$ pc (Reipurth 1989; Reipurth et al. 1997). In this Letter, we examine the properties of warm molecular gas along the jet feature with IRAC images. We also study the molecular outflow and the embedded YSO associated with the jet using, respectively, $\mathrm{CO}$ data and a set of infrared through millimeter photometric data.

\section{OBSERVATIONS}

The Spitzer c2d Legacy Program observed core A of L1251 (L1251-A) at 3.6, 4.5, 5.8, and $8.0 \mu \mathrm{m}$ with IRAC (Fazio et al. 2004) on 2004 September 4 and at 24 and $70 \mu \mathrm{m}$ with the Multiband Imaging Photometer for Spitzer (MIPS; Rieke et al. 2004) on 2004 November 30 (PID:139; AOR keys: IRAC 5165824, and MIPS 9425664). The detailed description of the data processing can be found in the c2d final delivery documentation (Evans et al. 2007).

To cover the whole region of the infrared jet, the CO 2-1 transition at $230.537970 \mathrm{GHz}$ has been mapped with the $6 \mathrm{~m}$ telescope at the Seoul National Radio Astronomical Observatory in 2009 March and April. The beam FWHM is $48^{\prime \prime}$ at $230 \mathrm{GHz}$. The main beam efficiency and pointing accuracy are 0.57 and $\sim 3^{\prime \prime}$, respectively. The velocity resolution is $0.127 \mathrm{~km} \mathrm{~s}^{-1}$ after binning by two channels.

For the analysis of the spectral energy distributions (SEDs), we also use $350 \mu \mathrm{m}$ and $1.2 \mathrm{~mm}$ continuum emission maps observed with the Submillimeter High Angular Resolution Camera II (SHARC-II) mounted on the Caltech Submillimeter Observatory, and the Max-Planck Millimeter Bolometer 


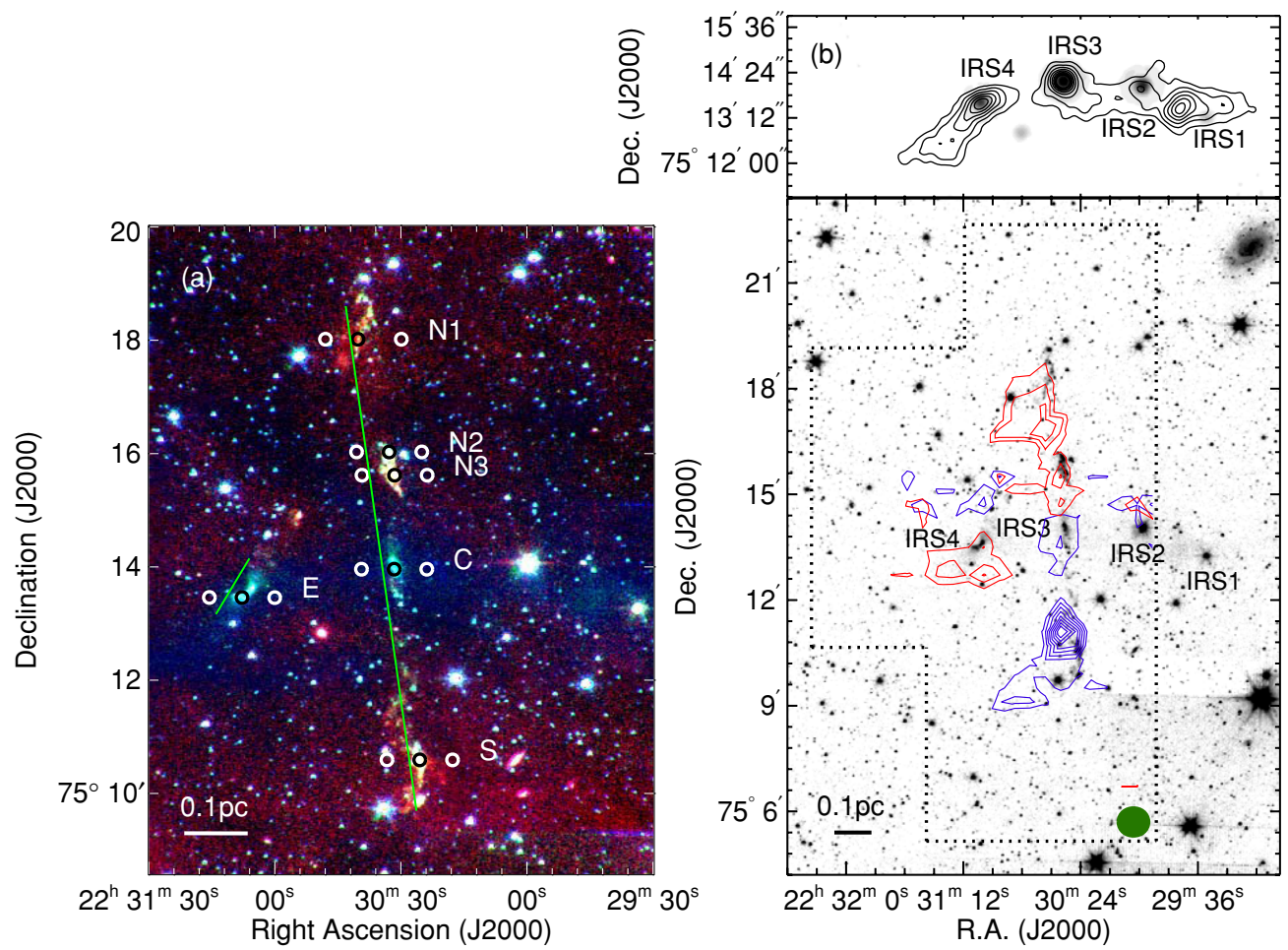

Figure 1. (a) Three-color composite Spitzer image of L1251-A. The IRAC 3.6, 4.5, and $8.0 \mu \mathrm{m}$ data are presented, respectively, as blue, green, and red. Green lines are placed along the jet and bipolar nebula features. Black circles show the regions where the colors plotted in Figure 3 were derived, and white circles to the east and west of each black circle indicate where the background was measured. (b) Upper panel: $1.2 \mathrm{~mm}$ MAMBO intensity map (contours) on top of MIPS $70 \mu \mathrm{m}$ image. IRS3 and IRS4 are located at the peaks of MAMBO emission. Another emission peak exists between IRS1 and IRS2, which might be a prestellar core. Lower panel: CO molecular outflow map (contors) on top of the IRAC $4.5 \mu \mathrm{m}$ image. The dotted line encloses the total area mapped, and the green filled circle at the right bottom denotes the beam at $230 \mathrm{GHz}$. The blue contours are integrated from -13.0 to $-7.0 \mathrm{~km} \mathrm{~s}^{-1}$, while the red contours are integrated from -1.0 to $5.0 \mathrm{~km} \mathrm{~s}{ }^{-1}$. The contours start at 1.2 and $1.6 \mathrm{~K} \mathrm{~km} \mathrm{~s}^{-1}$ for blue and red components, respectively, and increase by $0.4 \mathrm{~K} \mathrm{~km} \mathrm{~s}^{-1}$. The lobes of the main outflow are well correlated with the infrared jet along the NS direction.

Table 1

Properties of YSO Candidates in L1251-A

\begin{tabular}{|c|c|c|c|c|c|c|c|c|c|c|c|c|c|}
\hline \multirow[t]{2}{*}{ YSOs } & \multirow[t]{2}{*}{ R.A. } & \multirow[t]{2}{*}{ Decl. } & \multicolumn{8}{|c|}{ Fluxes (mJy) } & \multirow[t]{2}{*}{$L_{\mathrm{bol}}\left(L_{\odot}\right)$} & \multirow[t]{2}{*}{$T_{\text {bol }}(\mathrm{K})$} & \multirow[t]{2}{*}{$L_{\mathrm{smm}} / L_{\mathrm{bol}}$} \\
\hline & & & $3.6 \mu \mathrm{m}$ & $4.5 \mu \mathrm{m}$ & $5.8 \mu \mathrm{m}$ & $8.0 \mu \mathrm{m}$ & $24 \mu \mathrm{m}$ & $70 \mu \mathrm{m}$ & $350 \mu \mathrm{m}^{\mathrm{a}}$ & $1.2 \mathrm{~mm}^{\mathrm{b}}$ & & & \\
\hline $\operatorname{IRS}{ }^{\mathrm{c}}$ & 222933.4 & 751315.9 & 5.62 & 10.50 & 15.60 & 18.20 & 55.8 & 150 &. & $\ldots$ & 0.07 & 290 & . \\
\hline IRS $2^{\mathrm{d}}$ & 222959.5 & 751403.2 & 15.80 & 21.80 & 25.20 & 27.30 & 272 & 848 & $\ldots$ & 713 & 0.4 & 230 & $\cdots$ \\
\hline $\mathrm{IRS}^{\mathrm{e}}$ & 223031.9 & 751408.8 & 0.13 & 0.42 & 0.32 & 0.16 & 4.97 & 1400 & 9400 & 1020 & 0.8 & 24 & 0.12 \\
\hline IRS $4^{\mathrm{f}}$ & 223105.6 & 751337.1 & 0.43 & 1.07 & 0.75 & 0.37 & 1.90 & 688 & 7500 & 925 & 0.6 & 21 & 0.13 \\
\hline
\end{tabular}

\footnotetext{
Notes.

a SHARCII aperture size $=40^{\prime \prime}$

b MAMBO aperture size $=80^{\prime \prime}$.

${ }^{\text {c }}$ SSTc2d J222933.4+751316.

${ }^{\mathrm{d}}$ SSTc2d J222959.5+751403 (IRAS 22290+7458).

e SSTc2d J223031.9+751409.

${ }^{\text {f }}$ SSTc2d J223105.6+751337.
}

(MAMBO) at the IRAM $30 \mathrm{~m}$ telescope, respectively. Details of these continuum data can be found in $\mathrm{Wu}$ et al. (2007) and Kauffmann et al. (2008).

\section{RESULTS}

Figure 1(a) shows a three-color image comprised of IRAC 3.6, 4.5, and $8.0 \mu \mathrm{m}$ images. The dominant feature in the image is a jet extending about $10^{\prime}$. The extended blue color emission perpendicular to the jet is consistent with the distribution of the millimeter dust continuum emission (see the upper panel of Figure 1(b)), so it is likely dominated by scattered light within a dense cloud with some contribution from shocked gas associated with jets and outflows. There is also a small bipolar nebula structure, similar to that seen in core E of L1251 (L1251B; Lee et al. 2006), to the east of the long jet.

In Figure 1(a), stellar objects (IRS3 and IRS4) associated with the jet and bipolar nebula are not clearly seen. However, these central engines of the jet and bipolar structure are well detected at 24 and $70 \mu \mathrm{m}$ (see Table 1 for fluxes). At $70 \mu \mathrm{m}$, the two infrared sources become brighter (see the upper panel of Figure 1(b)). In addition, as seen in Figure 1(b), these sources are each located at both $350 \mu \mathrm{m}$ and $1.2 \mathrm{~mm}$ dust continuum peaks, indicating they are likely very embedded objects. In L1251A, the c2d team identified four YSOs, marked as IRS1, IRS2, 


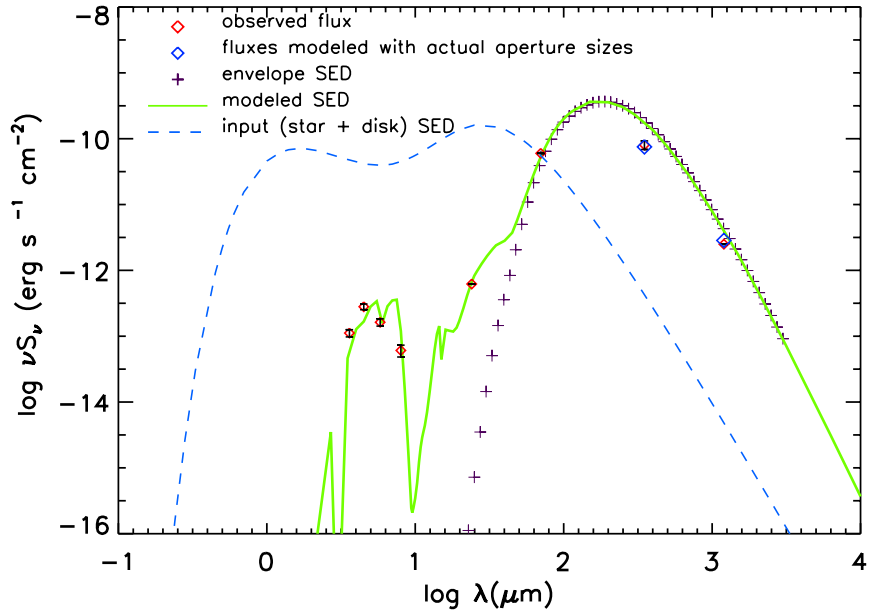

Figure 2. Best-fit model SED (green solid line) to the observed fluxes (red diamonds) of IRS3, with the internal input SED shown (blue dashed line). Blue diamonds represent model fluxes in the apertures used for photometry. Crosses indicates the SED emerging only from the envelope. This SED modeling gives an internal luminosity for IRS3, produced mostly by accretion, of $\sim 0.9 L_{\odot}$.

IRS3, and IRS4 in Figure 1(b). The infrared source between IRS3 and IRS4 is classified as a galaxy based on the c $2 \mathrm{~d}$ criteria (Evans et al. 2007). Table 1 lists fluxes of the four YSOs at various wavelengths, and the SED of the YSO associated with the jet (IRS3) is shown in Figure 2 (as red diamonds). The SED clearly indicates that IRS3 has a very thick envelope with strong (sub)millimeter emission. IRS4 is associated with the bipolar nebula and shows a similar SED to that of IRS3, although it is not presented in this Letter.

Figure 1(b) (lower panel) shows the CO 2-1 outflow map on top of the IRAC $4.5 \mu \mathrm{m}$ image. The outflow map covers the whole jet feature as well as the bipolar nebula. Along the jet, it shows two distinct lobes with little overlap, suggesting a large inclination of rotational axis with respect to the line of sight. The outflow lobes are well correlated spatially with the infrared jet. No clear outflow structure is seen toward IRS4 and its associated bipolar nebula, although some weak redshifted emission is present.

\section{ANALYSIS}

The jet feature associated with IRS3 appears consistently in all IRAC bands, indicating that the jet emission is probably produced by a common gas component such as molecular hydrogen, which has its line transitions distributed over all IRAC bands. Therefore, we calculate the IRAC colors of [5.6 $\mu \mathrm{m}] /$ [8.0 $\mu \mathrm{m}]$ and $[4.5 \mu \mathrm{m}] /[8.0 \mu \mathrm{m}]$ to compare with those of thermal gas modeled to explain the molecular hydrogen emission from the jet (Figure 3 ). The colors were calculated in 4 pixel $\left(\sim 5^{\prime \prime}\right)$ radius apertures at the positions marked with black circles in Figure 2. In the model calculation of the colors (Figure 3), the molecular gas was assumed to be composed of $\mathrm{H}_{2}$ and $\mathrm{He}$, with $n(\mathrm{He})=0.2 n\left(\mathrm{H}_{2}\right)$. We calculated the level population of $\mathrm{H}_{2}$ gas in statistical equilibrium. The collisional coefficients were obtained from Le Bourlot et al. (1999), and an ortho-to-para ratio of 3.0 is assumed. The temperature of the shocked $\mathrm{H}_{2}$ gas was modeled in two ways: isothermal and power-law admixed. In the latter, the infinitesimal $\mathrm{H}_{2}$ column density has a power-law relation with the temperature $T, d N \sim T^{-b} d T$, in the range of 300-4000 K. More detailed descriptions can be found in Neufeld \& Yuan (2008) and Shinn et al. (2009a, 2009b).

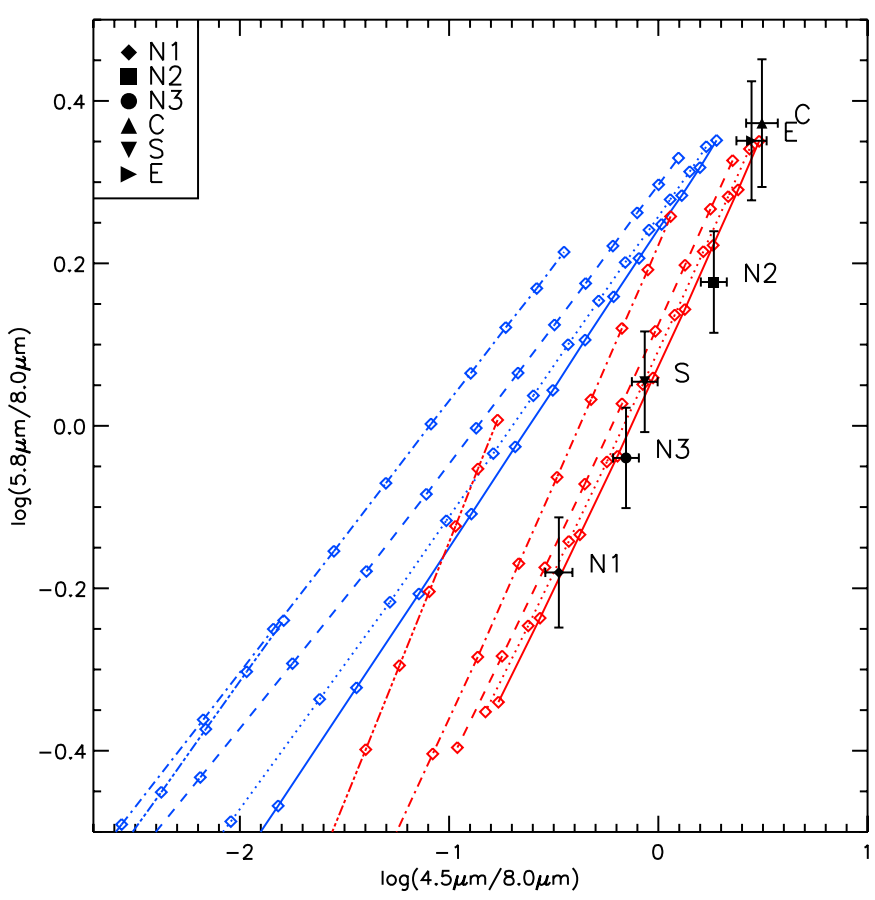

Figure 3. Two-color diagram of $[4.5 \mu \mathrm{m}] /[8.0 \mu \mathrm{m}]$ and $[5.6 \mu \mathrm{m}] /[8.0 \mu \mathrm{m}]$ expected from pure $\mathrm{H}_{2}$ emission with an assumption of $A_{v}=5 \mathrm{mag}$. Blue lines: single-component models for $n\left(\mathrm{H}_{2}\right)=10^{4}$ (dot-dot-dot-dashed), $10^{5}$ (dot-dashed), $10^{6}$ (dashed), $10^{7} \mathrm{~cm}^{-3}$ (dotted), and LTE (solid). Open squares represent the results of a range of temperatures up to $2000 \mathrm{~K}$ (top right of each curve) in steps of $100 \mathrm{~K}$. Red lines: models with a power-law temperature distribution, with $d N=a T^{-b} d T$ and $b$ in the range from 2 (top right of each curve) to 6 . The line type for each model is the same as in the single-component models. Each symbol represents the color measured at each position marked in Figure 1(a). The error bars include the flux measurement error and the flux calibration error $(\sim 10 \%)$.

We know that this jet is located inside a molecular cloud (Sato et al. 1994); thus, the extinction close to the jet engine is possibly much higher than $0.5 \mathrm{mag}$, which is the interstellar extinction toward L1251-A (Kun \& Prusti 1993). Therefore, the expected colors are extinction corrected with $A_{V} \sim 5 \mathrm{mag}$, which is obtained from the $1.2 \mathrm{~mm}$ dust continuum emission at 0.1 pc from IRS3, following the "Milky Way, $R_{V}=3.1$ " curve (Weingartner \& Draine 2001; Draine 2003). Extinctioncorrected colors with $A_{V} \sim 0.5$ and 5 mag are not different. However, close to YSOs (C and E in Figure 1(a)), $A_{V} \sim 50 \mathrm{mag}$. We tested this high extinction only for the colors of $\mathrm{C}$ and $\mathrm{E}$, which move diagonally up and right in the color-color diagram.

According to the comparison between the observed and modeled colors along the jet, the IRAC colors can be explained with the power-law admixture model, as Neufeld \& Yuan (2008) and Shinn et al. (2009a, 2009b) found. All colors are located between the model of $n\left(\mathrm{H}_{2}\right)=10^{6} \mathrm{~cm}^{-3}$ and the LTE line, where the pre-shock density must be higher than $10^{5} \mathrm{~cm}^{-3}$ (Wilgenbus et al. 2000). There might be some contribution from other emission components not considered in our model, such as the CO $v=1-0$ transitions in IRAC 2. However, such contamination is not likely according to Neufeld \& Yuan (2008). The power-law index, $b$, is the smallest $(\sim 2)$ at the jet feature close to the central embedded source ( $\mathrm{C}$ in Figure 1(a)) and the bipolar nebula feature ( $E$ in Figure $1(a)$ ) in the east of the long jet, indicating that there are more hot components close to the embedded YSOs. Alternatively, the scattered light in the outflow cavity may enhance the colors (more scattered light at shorter wavelengths). If we apply $A_{V}=50 \mathrm{mag}$, the colors at C 
Table 2

Outflow and Mass Accretion Properties

\begin{tabular}{|c|c|c|}
\hline Quantity & Units & Value \\
\hline Outflow momentum flux $\left(F_{\mathrm{C} O}\right)$ & $10^{-6} M_{\odot} \mathrm{km} \mathrm{s}^{-1} \mathrm{yr}^{-1}$ & $\geqslant 5.9 \frac{\sin i}{\cos ^{2} i} a$ \\
\hline Average protostellar mass accretion rate $\left(\left\langle\dot{M}_{\mathrm{a} c c}\right\rangle\right)$ & $10^{-6} M_{\odot} \mathrm{yr}^{-1}$ & $\geqslant 1.6 \frac{\cos i}{\sin i}$ \\
\hline Dynamical time $\left(\tau_{\mathrm{d}}\right)$ & $10^{4} \mathrm{yr}$ & $\geqslant 14.2 \frac{\cos i}{\sin i}$ \\
\hline Protostellar mass accreted $\left(M_{\mathrm{acc}}\right)$ & $M_{\odot}$ & $\geqslant 0.22 \frac{1}{\cos i}$ \\
\hline Accretion luminosity $\left(L_{\mathrm{acc}}\right)$ & $L_{\odot}$ & $\geqslant 3.6 \frac{\sin i}{\cos ^{3} i}$ \\
\hline
\end{tabular}

Note. ${ }^{\mathrm{a}} i$ is the inclination of the rotational axis with respect to the line of sight.

and E become similar to those of the model with $b \sim 1$. Except for these two points, $b$ is between 3.3 and 5.2, consistent with the results of Neufeld \& Yuan (2008), who showed that this range of $b$ values can be explained by the paraboloidal bow shock model. Bow shocks can be considered as a geometrical summation of planar C-shocks, whose post-shock temperature is nearly isothermal (Neufeld et al. 2006). In this sense, Neufeld \& Yuan (2008) showed that a single bow shock generates a shocked $\mathrm{H}_{2}$ gas with $b \sim 3.6$, and $b$ can be higher than 3.8 when slow bow shocks, where $\mathrm{H}_{2}$ is not dissociated even at the apex, are mixed with fast bow shocks.

Following Young et al. (2004), Bourke et al. (2006), and Dunham et al. (2006), we modeled the SED of the YSO associated with the jet (IRS3) using the one-dimensional (1D) dust continuum radiative transfer code DUSTY (Ivezic et al. 1999). In addition to a central stellar object, a 1D disk model (Butner et al. 1994) was adopted with a surface density profile of $\Sigma(r) \propto r^{-1.5}$ and temperature profile of $T(r) \propto r^{-0.35}$ to calculate the internal input SED for the best-fit model. The envelope is assumed to have a power-law density profile and is heated by the interstellar radiation field attenuated by $A_{V}=$ 3 mag. For the dust opacity, we adopt the same dust model used by Crapsi et al. (2008): a mixture of carbon (29\% of the total dust mass) and silicate grains covered with various molecular ices. For a detailed explanation of the modeling procedure, see Young \& Evans (2005) and Dunham et al. (2006). The best-fit SED to the observed fluxes is presented in Figure 2. The best-fit model has an internal luminosity of $\sim 0.9 L_{\odot}$ with $T_{\text {star }}=2500 \mathrm{~K}$. This internal luminosity derived by the model, which is considered to be the true bolometric luminosity of the source, is greater than $L_{\mathrm{bol}}$ calculated from observed fluxes because some of these long-wavelength fluxes were measured with apertures smaller than the total extent of the source. The envelope of the model has a power-law density profile index of $p=1.55$, a mass of $\sim 12 M_{\odot}$, and inner and outer radii of 250 and 18,000 AU, respectively. The internal luminosity derived by the model can be considered the current accretion luminosity.

The average accretion luminosity over the YSO lifetime can be derived from the momentum flux in the outflow. We use the CO $J=2-1$ map to calculate the outflow momentum flux, $F_{\mathrm{CO}}$, following the method presented by Hatchell et al. (2007). This quantity is the average rate at which momentum is injected into the outflow, and we calculate $F_{\mathrm{CO}} \geqslant 5.9 \times 10^{-6}$ $\frac{\sin i}{\cos ^{2} i} M_{\odot} \mathrm{km} \mathrm{s}^{-1} \mathrm{yr}^{-1}$ and list this result in Table 2. There is an inclination dependence that we leave in the result since the source inclination is not known. Two additional unknowns in the above calculation are the optical depth and the temperature of the outflowing gas (needed to convert line intensity to column density assuming LTE). Following M. Dunham et al. (2010, in preparation), we assume the gas is optically thin and has a temperature of $17.6 \mathrm{~K}$, which minimizes the line intensity to column density conversion. Optically thick gas and any other temperature in the range of $10-100 \mathrm{~K}$ will increase $F_{\mathrm{CO}}$, thus the above result is a very strong lower limit to the true value.

Since molecular outflows are driven by the transfer of momentum from a jet/wind ejected by the protostellar system to the ambient medium, and the jet/wind ejection process is closely tied to accretion onto the protostar, the calculated $F_{\mathrm{CO}}$, which represents the average rate at which momentum is injected into the outflow, is related to $\left\langle\dot{M}_{\text {acc }}\right\rangle$, the time-averaged protostellar mass accretion rate (see Bontemps et al. 1996):

$$
\left\langle\dot{M}_{\mathrm{acc}}\right\rangle=\frac{1}{f_{\mathrm{ent}}} \frac{\dot{M}_{\mathrm{acc}}}{\dot{M}_{w}} \frac{1}{V_{w}} F_{\mathrm{CO}} .
$$

In the above equation, $\dot{M}_{w}$ is the mass-loss rate in the jet/ wind, $V_{w}$ is the velocity of the jet/wind, and $f_{\text {ent }}$ is the entrainment efficiency between the jet/wind and the ambient gas. With typical values for the above parameters of $\frac{\dot{M}_{w}}{\dot{M}_{\text {acc }}}=0.1, V_{w} \sim$ $150 \mathrm{~km} \mathrm{~s}^{-1}$, and $f_{\text {ent }}=0.25$ (Bontemps et al. 1996, and references therein), we calculate $\left\langle\dot{M}_{\mathrm{acc}}\right\rangle \geqslant 1.6 \times 10^{-6} \frac{\sin i}{\cos ^{2} i} M_{\odot} \mathrm{yr}^{-1}$. An outflow dynamical time $\left(\tau_{d} \geqslant 14.2 \times 10^{4} \frac{\cos i}{\sin i} \mathrm{yr}\right)$ is calculated by dividing the extent of the outflow $\left(400^{\prime \prime} / \sin i\right)$ by the outflow velocity $\left(\sim 4 / \cos i \mathrm{~km} \mathrm{~s}^{-1}\right)$. Assuming accretion at the above rate for this length of time gives an accreted protostellar mass of $M_{\text {acc }} \geqslant 0.22 \frac{1}{\cos i} M_{\odot}$. Finally, accretion at the above rate onto a protostar with this mass and $R=3 R_{\odot}$ gives a spherical accretion luminosity of $L_{\text {acc }}=\frac{G M\left\langle\dot{M}_{\text {acc }}\right\rangle}{R} \geqslant 3.6 \frac{\sin i}{\cos ^{3} i} L_{\odot}$.

\section{DISCUSSION}

We calculated the bolometric luminosity and temperature of each YSO identified in L1251-A, following the method used in Dunham et al. (2008) (see Table 1). The apparent drivers (IRS3 and IRS4) of the infrared jet and bipolar nebula have $L_{\text {bol }} \leqslant 1 L_{\odot}$, similar to IRS2 in L1251B (L1251B-IRS2), another source associated with infrared bipolar structure (Lee et al. 2006). Although IRS3 and IRS4 are $\sim 10$ times fainter than L1251B-IRS2 at $24 \mu \mathrm{m}$, they are very bright at wavelengths longer than $70 \mu \mathrm{m}$. Their bolometric temperatures are $\sim 20 \mathrm{~K}$, much lower than that of L1251B-IRS2 (140 K). Finally, the ratio of $L_{\mathrm{smm}} / L_{\text {bol }}$ for IRS3 and IRS4 ( $\sim 0.13$ and 0.17 , respectively, where $L_{\mathrm{smm}}$ is the luminosity at $\left.\lambda>350 \mu \mathrm{m}\right)$ is much greater than that of L1251B-IRS2 ( $\sim 0.03)$, indicative of Class 0 sources with very thick envelopes.

The dominant feature in L1251-A is the long, well-collimated jet associated with IRS3. This type of jet structure can be produced in the IRAC bands by rovibrational or pure rotational line emission of $\mathrm{H}_{2}$ in a pulsating jet with little precession (model A1 in Smith \& Rosen 2005). According to Smith \& Rosen (2005), 
this jet model (A1) transfers the bulk kinetic energy to large distances but excites $\mathrm{H}_{2}$ with the lowest efficiency. However, the jet shows undulating structure toward both ends. Smith \& Rosen (2005) showed this kind of helical stream in a jet with a slow uniform-speed precession. Therefore, the jet in L1251-A might be explained with a small precession angle (but greater than that of A1) and a long pulsating period (much longer than 60 years). In fact, the jet associated with the northern, red component of the $\mathrm{CO}$ outflow features two distinct emission bulks, indicative of episodic ejection. The time interval between episodes of ejection is $1300 / \sin i$ years if we assume a jet speed of $150 \mathrm{~km} \mathrm{~s}^{-1}$.

The infrared jet and the $\mathrm{CO}$ outflow are well correlated in shape and length as seen in Figure 1(b). From the CO 2-1 map, we calculated a time-averaged mass accretion rate and the accretion luminosity expected from accretion at this rate (Table 2). In spite of uncertainties in $f_{\text {ent }}$ and $\dot{M}_{w} / \dot{M}_{\text {acc }}$, the calculated time-averaged accretion rate (and resulting accretion luminosity) are strong lower limits, as explained in Section 4. In addition, a large inclination (there is little overlap between red and blue components of the $\mathrm{CO}$ outflow and no significant intensity difference between two infrared jet lobes) of $70^{\circ}$ will give $L_{\text {acc }} \sim 83 L_{\odot}$, which is much greater than $L_{\text {acc }}$ derived from dust modeling $\left(0.9 L_{\odot}\right)$. Since the luminosity from dust modeling depends on the current mass accretion rate, this rate must be lower now than the time-averaged value over the outflow lifetime.

The comparison between the observed IRAC colors and the ones from the model of a power-law admixture of gas (see Figure 3) indicates the amount of hot components varies (in general, more hot gas closer to the jet engine). If we use the same colors ([3.6]-[4.5] versus [4.5]-[5.8]) as those presented by Ybarra \& Lada (2009), who modeled only the hot (2000-4000 K) gas component, $\mathrm{C}$ and $\mathrm{E}(b \sim 2$, see Figure 3$)$ are located at $n(\mathrm{H})>10^{5} \mathrm{~cm}^{-3}$, while $\mathrm{N} 2, \mathrm{~N} 3$, and $\mathrm{S}(b \sim 3.3-4.5)$ lie close to the model line of $n(\mathrm{H})=10^{5} \mathrm{~cm}^{-3}$. Therefore, the density inferred by their model is not very different from that inferred from our model, although, unlike in our model, Ybarra \& Lada (2009) included collisions between $\mathrm{H}_{2}$ and $\mathrm{H}$. However, N1 is placed at $n(\mathrm{H}) \sim 2 \times 10^{3} \mathrm{~cm}^{-3}$ in their model, consistent with our model result since the model of Ybarra \& Lada (2009) considered only the hot gas component, and in our model, $b \sim 5.2$ for N1 suggests a relatively small amount of hot gas at that position.

We acknowledge the support by the National Research Foundation of Korea (NRF) grant funded by the Korean govern- ment (MEST) (No. 2009-0062865). This work was also supported in part by a grant (R01-2007-000-20336-0) from the Basic Research Program of the NRF. Support for this work, part of the Spitzer Legacy Science Program, was provided by NASA through contract 1224608 issued by the Jet Propulsion Laboratory, California Institute of Technology, under NASA contract 1407.

\section{REFERENCES}

Bontemps, S., André, P., Terebey, S., \& Cabrit, S. 1996, A\&A, 311, 858

Bourke, T. L., et al. 2006, ApJ, 649, L37

Butner, H. M., Natta, A., \& Evans, N. J., II, 1994, ApJ, 420, 326

Crapsi, A., van Dishoeck, E. F., Hogerheijde, M. R., Pontoppidan, K. M., \& Dullemond, C. P. 2008, A\&A, 486, 245

Draine, B. T. 2003, ARA\&A, 41, 241

Dunham, M. M., Crapsi, A., Evans, N. J. II, Bourke, T. L., Huard, T. L., Mypers, P. C., \& Kauffmann, J. 2008, ApJS, 179, 249

Dunham, M. M., et al. 2006, ApJ, 651, 945

Evans, N. J., II, et al. 2003, PASP, 115, 965

Evans, N.J., II, et al. 2007, Final Delivery of Data from the c2d Legacy Project: IRAC and MIPS (Pasadena CA: SSC)

Fazio, G. G., et al. 2004, ApJS, 154, 10

Fukui, Y. 1989, in ESO Conf. Workshop Proc., 33, Low Mass Star Formation and Pre-Main Sequence Objects, ed. B. Reipurth (Garching: ESO), 95

Hatchell, J., Fuller, G. A., \& Richer, J. S. 2007, A\&A, 472, 187

Ivezic, A., Neukova, M., \& Elitzur, M. 1999, User Manual for DUSTY (Lexington, KY: Univ. Kentucky)

Kauffmann, J., Bertoldi, F., Bourke, T. L., Evans, N. J., II, \& Lee, C. W. 2008, A\&A, 487, 993

Kun, M., \& Prusti, T. 1993, A\&A, 272, 235

Le Bourlot, J., Pineau des Forêts, G., \& Flower, D. R. 1999, MNRAS, 305, 802

Lee, J.-E., et al. 2006, ApJ, 648, 491

Maret, S., et al. 2009, ApJ, 698, 1244

Morgan, J. A., Schloerb, F. P., Snell, R. L., \& Bally, J. 1991, ApJ, 376, 618

Myers, P. C., Adams, F. C., Chen, H., \& Schaff, E. 1998, ApJ, 492, 703

Neufeld, D. A., \& Yuan, Y. 2008, ApJ, 678, 974

Neufeld, D. A., et al. 2006, ApJ, 649, 816

Reipurth, B. 1989, Nature, 340, 42

Reipurth, B., Bally, J., \& Devine, D. 1997, AJ, 114, 2708

Rieke, G. H., et al. 2004, ApJS, 154, 25

Sato, F., Mizuno, A., Nagahama, T., Onishi, T., Yonecura, Y., \& Fukui, Y. 1994, 435,279

Shinn, J.-H., Koo, B.-C., Burton, M. G., Lee, H.-G., \& Moon, D.-S. 2009a, ApJ, 693, 1883

Shinn, J.-H., Koo, B.-C., Burton, M. G., Lee, H.-G., \& Moon, D.-S. 2009b, Adv. Space Res., in press (arXiv:0908.4311)

Smith, M. D., \& Rosen, A. 2005, MNRAS, 357, 1370

Weingartner, J. C., \& Draine, B. T. 2001, ApJ, 548, 296

Wilgenbus, D., Cabrit, S., Pineau des Forêts, G., \& Flower, D. R. 2000, A\&A, 356,1010

Wu, J., Dunham, M. M., Evans, N. J., II, Bourke, T. L., \& Young, C. H. 2007, AJ, 133,1560

Ybarra, J. E., \& Lada, E. A. 2009, ApJL, 695, 120

Young, C. H., \& Evans, N. J., II. 2005, ApJ, 627, 293

Young, C. H., et al. 2004, ApJS, 154, 396 